\title{
Contrôle hormonal, spécificité tissulaire et développement : des sites régulateurs communs
}

La régulation des gènes au cours du développement peut être corrélée, dans certains cas, à des perturbations physiologiques de l'organisme. Par exemple, de nombreux gènes métaboliques sont induits ou réprimés à la naissance ou au sevrage et ces modulations sont associées à de profonds changements au niveau hormonal et nutritionnel. L'action des glucocorticoïdes et du glucagon sur les enzymes de la néoglucogenèse illustre bien ce type de régulation. En effet, l'hypoglycémie néonatale entraîne une augmentation rapide de ces hormones. Cela se traduit par une induction d'enzymes telles que les transaminases, la phosphoénolpyruvate carboxykinase (PEPCK) et d'autres enzymes du métabolisme des acides aminés et de la néoglucogenèse. Le rôle primordial du glucagon et des glucocorticoïdes est reflété par l'induction prématurée de ces enzymes lorsque les hormones sont administrées in utero. Bien entendu, cette observation ne prouve pas que l'effet hormonal soit le seul élément déterminant dans l'activation de ces gènes à la naissance ; il est probable que d'autres facteurs liés au stade de développement et au tissu interviennent. Des études récentes ont établi que ces différents facteurs dépendant des hormones ou du développement pourraient interagir, directement ou indirectement, avec la même séquence d'ADN pour contrôler l'expression d'un gène codant pour une enzyme hépatique, la tyrosine aminotransférase (TAT) [1, 2 et réfé-

La mutation albinos chez la souris constitue l'un des modèles qui ont permis l'étude de la différenciation hépatique. Dans ces mutants, les gènes hépatiques, normalement activés au moment de la naissance, restent exprimés à un niveau bas, ce qui entraîne une léthalité périnatale. L'origine de la mutation est une délétion importante sur le chromosome 7. Les gènes dont l'expression n'est pas activée sont nombreux, les plus étudiés étant la TAT et de la PEPCK. L'équipe de G. Schütz (Heidelberg, RFA) a réalisé un clonage par soustraction des ADNc correspondant aux messages présents chez la souris normale et absents chez les mutants [1]. Une quinzaine d'ARNm clonés ont été analysés; certains de ces messagers ont une expression purement hépatique et sont, comme prévu, absents dans les souris mutantes. D'autres, comme le messager de l'arginine succinate synthétase, ont une expression ubiquitaire chez les souris normales et mutantes. Pour ces gènes-là, l'anomalie porte uniquement sur l'expression hépatique. La quasi-totalité des gènes étudiés est induite par les glucocorticoïdes et l'AMPc dans le foie, à l'exception notable du gène du facteur transcriptionnel C/EBP $(\mathrm{m} / \mathrm{s}$, $n^{\circ} 5$, vol. 6, p. 486). Ces études établissent ainsi une corrélation étroite entre l'activation de gènes dans le foie à la naissance et leur régulation par l'AMPc et les glucocorticoïdes, démontrant ainsi la convergence des deux phénomènes.

Une autre approche génétique utili- sant la technique des hybrides somatiques a permis d'aborder un aspect différent du contrôle des gènes hépatiques. En effet, un hybride entre une cellule d'hépatome différenciée et un fibroblaste n'exprime pas les fonctions hépatiques. Ces observations ont été interprétées comme reflétant la présence de loci génétiques (dont un sur le chromosome 17 murin, Tsc-1) qui répriment l'expression de certains gènes spécifiques du foie comme celui de la TAT et la PEPCK. Cependant le groupe de gènes contrôlés par Tse 1 ne recouvre que partiellement celui des gènes dont l'expression est modifiée par la mutation albinos. L'action de l'AMPc et des glucocorticoïdes reste néanmoins fondamentale, puisque dans des hybrides contenant le locus Tse 1, ces hormones parviennent à lever la répression de certains gènes hépatiques dont l'expression se rapproche ainsi du niveau normal [1]. Il semble donc que l'action du produit du gène Tse 1 converge à un certain moment avec l'action hormonale. L'étude des séquences régulatrices du gène de la TAT a permis de mettre en évidence un site particulièrement intéressant à environ - 3,5 kilopaires de bases (kpb) de l'initiation de la transcription. Ce site d'environ $100 \mathrm{pb}$, est constitué de deux séquences cibles probablement reconnues par des protéines différentes mais agissant en synergie [2]. L'un des domaines, BIII, contribue à la spécificité tissulaire de l'expression de la TAT et l'autre, BI, présente de fortes homologies avec la séquence res- 
ponsable des effets de l'AMPc (voir figure 1). Cette deuxième séquence est reconnue par une protéine nucléaire présente uniquement dans les cellules d'hépatomes différenciées. De plus, dans les hybrides contenant le locus Tse 1, cette liaison disparaît et ne réapparaît qu'après traitement par l'AMPc. L'occupation de ces sites est corrélée à l'expression et à la régulation du gène de la TAT. Ces résultats illustrent clairement l'antagonisme entre l'action de l'AMPc et les effets du gène Tse 1 . Ce gène ne code probablement pas pour une protéine de liaison à l'ADN puisque son action se traduit par l'altération de la liaison d'un autre facteur sans apparition d'une nouvelle interaction protéine-ADN. Son véritable rôle reste à élucider. Son action pourrait se traduire par une répression de gènes hépatiques dans les tissus autres que le foie. Cette répression serait contrebalancée par la synergie de facteurs spécifiques du foie et de facteurs sensibles au traitement hormonal ; c'est justement cette situation qui est retrouvée dans le foie du nouveau-né.

La participation à la spécificité tissulaire d'un site sensible à l'AMPc a déjà été mise en évidence dans le promoteur du gène de la sous-unité $\alpha$ des hormones glycoprotéiques humaines (HCG, FSH, LH, TSH) [3]. Dans ce système, une séquence responsable de l'expression "tissu spécifique " de l'hormone n'est active qu'en présence de la séquence CRE (cyclic AMP response element). Ainsi la spécificité tissulaire nécessite une synergie entre un facteur spécifique de tissu et un facteur plus répandu et sensible à l'AMPc. Il faut cependant noter que la séquence CRE n'est pas seulement nécessaire à l'activation du promoteur par l'AMPc, mais est aussi requise pour l'expression basale du promoteur. La même observation a été faite pour la séquence CRE du promoteur du gène de la phosphoénolpyruvate carboxykinase [4]. La diversité des fonctions de cette séquence est à rapprocher de l'existence d'une famille de facteurs transcriptionnels capables de s'y lier [5]. Ces facteurs transcrip$\mathrm{m} / \mathrm{s} n^{\circ} 7$ vol. 6 , septembre 90

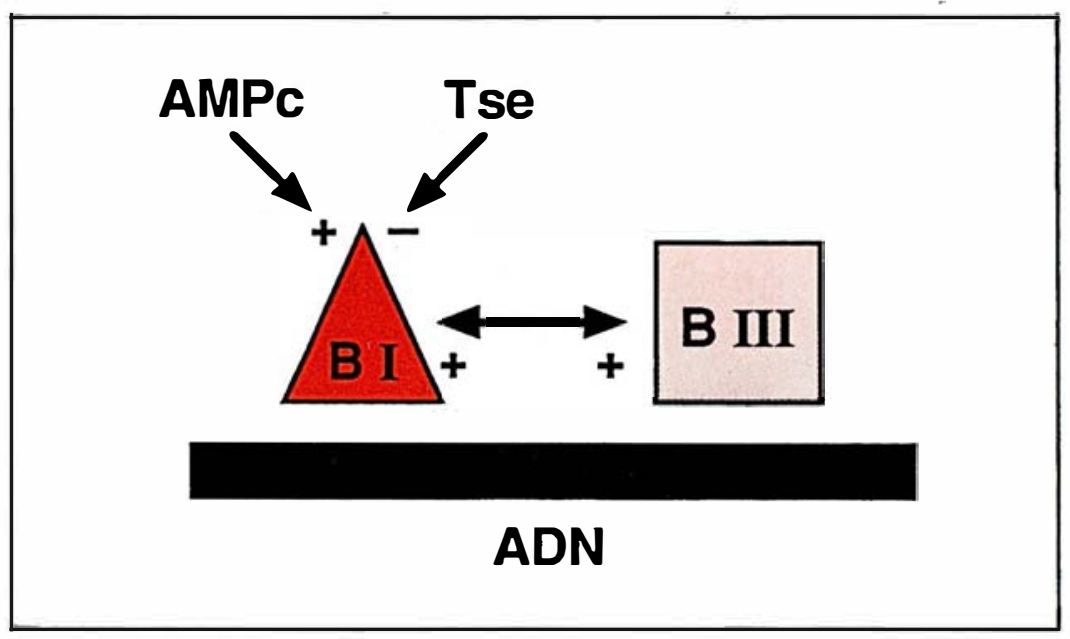

Figure 1. Le site régulateur du gène de la tyrosine aminotransférase : le modèle schématisé ci-dessus prévoit la liaison à cette séquence d'au moins deux protéines agissant en synergie : la protéine BIII, responsable de la spécificité cellulaire et la protéine BI qui se fixe au CRE. La fonction du Bl est activée par I'AMPc et inhibée par le produit du locus Tse.

tionnels sont retrouvés sous forme d'homodimères ou d'hétérodimères, ce qui augmente le nombre de combinaisons possibles. Cette famille de protéines se liant à la séquence CRE rappelle à plus d'un titre la famille des proto-oncogènes $c$-jun et $c$-fos [5], d'autant plus que ceux-ci peuvent parfaitement participer aux complexes se fixant sur les éléments CRE [6].

Si l'ensemble de ces observations permet de mieux comprendre la spécificité tissulaire de l'expression de certaines protéines, aucun des gènes mutés ou des gènes "répresseurs" n'a été isolé. C'est lorsque ces gènes seront clonés qu'on pourra vérifier leur mode d'action et déterminer à quels niveaux ils agissent. Se posera ensuite le problème de leur propre régulation! Il n'en demeure pas moins que ces études démontrent, sur un plan moléculaire, la relation étroite entre le contrôle hormonal et la spécificité tissulaire de certains gènes

\section{Robert Barouki}

Inserm U. 99, Hôpital Henri-Mondor, 51, avenue du Maréchal-de-Lattre-deTassigny, 94010 Créteil, France.

\section{RÉFÉRENCES}

1. Ruppert S, Boshart M, Bosch F, et al. Two genetically defined trans-acting loci coordinately regulate overlapping sets of liver-specific genes. Cell 1990; 61 :895-904. 2. Boshart M, Weih F, Schmidt A, et al. A cyclic AMP response element mediates repression of tyrosine aminotransferase gene transcription by the tissue-specific extinguisher locus Tse 1. Cell 1990: 61: 905-16. 3. Delegeane AM, Ferland LH, Mellon PL. Tissue specific enhancer of the human glycoprotein hormone $\alpha$-subunit gene : dependence on cyclic AMP-inducible ele ments. Mol Cell Biol 1987; 7 : 3994-4002. 4. Quinn PG, Wong TW, Magnusson MA, et al. Identification of basal and cyclic AMP regulatory elements in the promoter of the phosphoenolpyruvate carboxykinase gene. Mol Cell Biol 1988; 8 : 3467-75. 5. Ziff EB. Transcription factors : a new family gathers at the cAMP response site TIG $1990 ; 6: 69-72$

6. Macgregor PF, Abate C, Curran T. Direct cloning of leucine zipper proteins. Jun binds cooperatively to the CRE with CRE-BP1. Oncogene 1990; 5: 451-8. 